\title{
Time-Dependent Behavior of a Graphite/Thermoplastic Composite and the Effects of Stress and Physical Aging
}

Authorized Reprint from Journal of Composities Technology \& Research JAN. 1995 CCopyright 1995

American Society for Testing and Materials, 100 Barr Harbor Drive, West Conshohocken, PA $19428-2959$

\begin{abstract}
REFERENCE: Gates, T. S. and Feldman, M., "Time-Dependent Behavior of a Graphite/Thermoplastic Composite and the Effects of Stress and Physical Aging," Journal of Composites Technology \& Research, JCTRER, Vol. 17, No. 1, January 1995, pp. 33-42.
\end{abstract}

\begin{abstract}
Experimental studies were performed to determine the effects of stress and physical aging on the matrix dominated timedependent properties of IM7/8320 composite. Isothermal tensile creep/ aging test techniques developed for polymers were adapted for testing of the composite material. Time-dependent transverse $\left(S_{22}\right)$ and shear $\left(S_{66}\right)$ compliance's for an orthotropic plate were found from shortterm creep compliance measurements at constant, sub- $T_{\mathrm{g}}$ temperatures. These compliance terms were shown to be affected by physical aging. Aging time shift factors and shift rates were found to be a function of temperature and applied stress.
\end{abstract}

KEXWORDS: physical aging, composite materials, viscoelasticity, creep compliance, superposition

Design and trade studies are currently under way in the commercial aircraft industry to assess the materials and structures required for the development of the next generation, supersonic transport. This vehicle, designated the High Speed Civil Transport (HSCT) and targeted to carry over 300 passengers at speeds in excess of mach 2, will have a useful lifetime of over 60000 flight hours. During a typical flight, skin temperatures may reach up to $200^{\circ} \mathrm{C}$. To meet the weight requirements imposed by such design criteria, polymer matrix composite (PMC) materials are being considered for both primary and secondary structures.

One potential difficulty associated with using PMCs in such a vehicle is the task of predicting the changes in material properties due to aging of the PMC after long-term exposure at temperature. These changes in the composite's strength and stiffness will be primarily due to changes in the mechanical properties of the matrix material. The aging of a polymer matrix may be due to some combination of physical aging, chemical aging, and damage accumulation. It is the intent of this report to consider the effects due to just the physical aging process. This type of aging, considered to be a thermoreversible process, will cause changes in mechanical properties brought about by the volume recovery in the polymer upon cooling from above the glass transition $\left(T_{g}\right)$ temperature. During aging, the polymer moves towards a state of equilibrium. This state of equilibrium, defined as the point of minimum volume change, is approached asymptotically.

${ }^{1}$ Research scientist, NASA Langley Research Center, Hampton, VA. ${ }^{2}$ Old Dominion University, Norfolk, VA.

(C) 1995 by the American Society for Testing and Materials
Physical aging in polymers is a well-known phenomenon. Struik [1] performed one of the most comprehensive studies of physical aging. In his work he conducted numerous studies on the effects of load and environment on the physical aging of a variety of polymer systems. More recently, Sullivan [2] and Hastie and Morris [3] used experimental techniques established for polymers and extended them to study physical aging in glass/epoxy and graphite/ thermoplastic continuous fiber PMCs. Their studies concentrated on using momentary creep tests to determine the principal compliance terms and aging shift rates for a laminated composite as a function of temperature and aging time. Testing was performed isothermally and within the linear viscoelastic range. Results from this work showed that physical aging directly influenced the shortand long-term creep compliance. In an other work, Chen et al. [4] studied the effects of aging on the toughness of graphite/ thermoplastic, continuous fiber PMCs. Chen found that parameters such as Mode I strain energy release rate, damage initiation force, and propagation energy decreased with increases in the aging temperature and time.

The objectives of this research were to experimentally measure short-term elevated temperature creep compliance in IM7/8320, a graphite/thermoplastic, and determine the effects of stress and physical aging on the matrix-dominated compliance. These studies may provide a source of durability analysis tools and accelerated test methods for HSCT materials development.

\section{Tensile Creep Compliance}

The experimental study centered on using momentary creep compliance tests to determine the aging behavior of the principal compliance terms for a laminated composite as a function of stress and temperature. Using a three-parameter model of creep compliance, aging shift factors and shift rates were investigated for three temperatures below the $T_{g}$. Isothermal, elevated temperature creep tests provided the short-term creep compliance data. To establish stress levels for the aging tests, short-term, isothermal creep tests were run at increasingly higher stresses. In addition, creep/recovery tests provided insights into the effects of short versus long recovery periods and the applicability of Boltzman's superposition principle [5]. Other tests examined the effects of stress on creep compliance while aging to equilibrium.

\section{Analytical Models}

The analytical modeling provided a means of describing changes due to physical aging in creep compliance of a laminated plate. 
For the laminates in this study, the usual axes' definitions for an orthotropic plate were used, for example, $1=$ along the fiber, $2=$ transverse to the fiber, $x=$ axial loading direction, $y=$ transverse to loading direction, and the angle between the 1,2 axes and the $x-y$ axes was measured clockwise.

The general case of a thin orthotropic plate was considered. For a state of plane stress parallel to the $x=y$ plane in an orthotropic solid, the constitutive relation can be given by

$$
\left\{\begin{array}{l}
\epsilon_{11} \\
\epsilon_{22} \\
\gamma_{12}
\end{array}\right\}=\left[\begin{array}{ccc}
S_{11} & S_{12} & 0 \\
S_{21} & S_{22} & 0 \\
0 & 0 & S_{66}
\end{array}\right]\left\{\begin{array}{l}
\sigma_{11} \\
\sigma_{22} \\
\sigma_{12}
\end{array}\right\}
$$

where $\epsilon$ and $\gamma$ are the strains, $S_{i j}$ is the compliance, and $\sigma$ is the stress.

This set of equations implies that there are four independent constants needed to characterize the material. These constants are the longitudinal and transverse compliance, $S_{11}$ and $S_{22}$ respectively, Poison's ratio $v_{12}$, and the shear compliance $S_{66}$. A primary assumption was that physical aging occurred only in the polymer-matrix constituent. On the basis of tests performed by Sullivan [2] and Hastie and Morris [3], an assumption was that $S_{22}$ and $S_{66}$ were the two compliance terms to be affected by physical aging.

Creep tests provided the means for assessing the time-dependent compliance changes in both the $S_{22}$ and $S_{66}$ terms. These creep tests were analyzed using linear viscoelastic theory and standard [1] sub- $T_{8}$ creep/aging data reduction procedures. The term linear viscoelastic behavior implies that the principles of proportionality and superposition were both met. These conditions were checked experimentally and the results are given in following sections. The authors recognize that the polymer-matrix material may exhibit nonlinearity, particularly at elevated temperatures. However, it was felt that the simplicity of linear viscoelasticity made it desirable enough to investigate the use of it as a initial modeling scheme.

For a short-term (less than $10 \mathrm{~h}$ ) creep test, the log compliance versus $\log$ test time data for both $S_{22}$ and $S_{66}$ tests will appear as shown in the example provided by Fig. 1. A three-parameter expression was chosen to model the test data

$$
S_{i j}(t)=S_{i j}^{o} e^{(t / \tau)^{\beta}}
$$

where $S_{i j}$ is the time-dependent creep compliance term from Eq $1, S_{i j}^{o}$ is the initial creep compliance, $t$ is time, $\tau$ is the characteristic

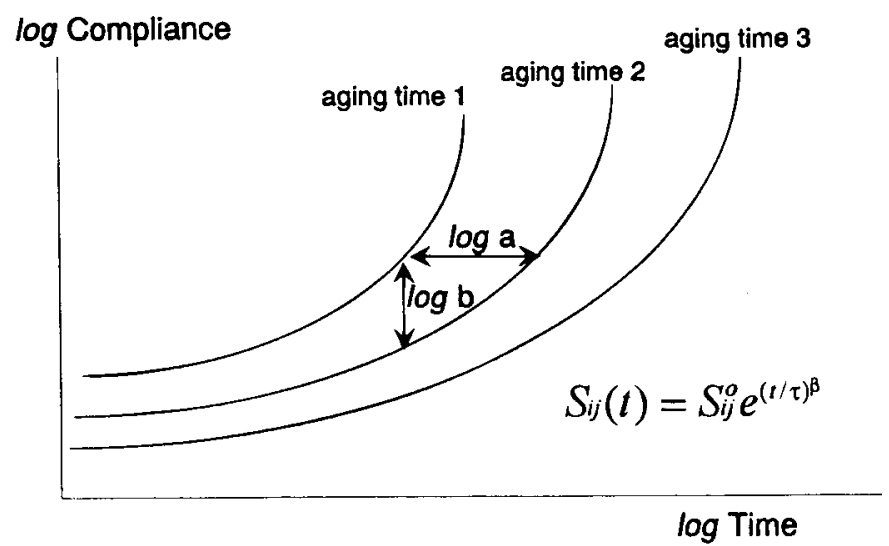

FIG. 1-Schematic of typical creep compliance data from a sequenced aging test. retardation time, and $\beta$ is the shape parameter. The fitting algorithm was based upon the Levenberg-Marquardt method [6]. Equation 2 has been used successfully by Struik [1] to model a wide range of polymers. The contribution of each parameter to the shape of the curve is well understood. For the continuous fiber-reinforced composite, the individual terms can be written

$$
\begin{aligned}
& S_{22}(t)=S_{22}^{o} e^{\left(t / \tau_{2}\right)^{\beta_{2}}} \\
& S_{66}(t)=S_{66}^{o} e^{\left(t / \tau_{6}\right)^{\beta_{6}}}
\end{aligned}
$$

The short-term creep compliance curves, as shown in Fig. 1, had a horizontal or time shift between curves defined by $\log$ a, and the vertical or initial compliance shift between curves defined as $\log$ b. Figure 1 schematically illustrates these shifts. It was assumed that both shifts were due to physical aging effects.

Once the time shifts $(\log$ a) were measured for a given set of compliance tests, the aging shift rate $(\mu)$ was calculated using

$$
\mu=\frac{d \log \left(a_{t e}\right)}{d \log \left(t_{e}\right)}
$$

where $t_{e}$ is the specific aging time for a given test. Struik [1] indicates that for sub- $T_{g}$ temperatures, the shift rates would be constant over the relatively short aging time of a test sequence and would have a value close to unity. By raising the test temperatures sufficiently close to $T_{g}$, Struik [1] and Lee and McKenna [7], found the shift rate of an aging test decreased significantly below unity. This was attributed to the material approaching an equilibrium state. The time required to reach this equilibrium state is dependent on the test temperature. Lee and McKenna [7] have stated that this equilibrium time was not dependent upon the state of stress. An empirical formula for estimating this equilibrium time $\left(t_{\infty}\right)$ for polymers was given by Struik [I] to be

$$
t_{\infty} \cong 100 e^{77\left(T_{g}-r\right)}
$$

where $T_{g}$ is the glass transition of the test material, $T$ is the test temperature in degrees $\mathrm{C}$, and $t_{\infty}$ is in seconds.

\section{Test Material and Specimen Configuration}

The composite material used in this study was a continuous carbon-fiber-reinforced amorphous thermoplastic fabricated by AMOCO and designated IM7/8320 (formally IM7/RadelX). Static lamina properties measured isothermally and given previously $[8$ are repeated for reference purposes in Table 1 . The $T_{g}$, as measurec by a differentiating scanning calorimeter (DSC), was $221.3^{\circ} \mathrm{C}$.

The static material property tests were run using a rectangular specimen geometry of approximately 24 by $2.5 \mathrm{~cm}$ with a 12- ol 8-ply thickness and a per ply thickness of approximately .013 . $\mathrm{cm}$. A set of thin end tabs were added to each end of the specimen For the creep tests, the transverse $\left(S_{22}\right)$ and shear $\left(S_{66}\right)$ complianct data came from $[90]_{12}$ and $[ \pm 45]_{25}$ specimens, respectively.

TABLE 1-Elastic material constants for unaged IM7/8320 [8].

\begin{tabular}{ccccc}
\hline Temperature, ${ }^{\circ} \mathrm{C}$ & $E_{1}, \mathrm{MPa}$ & $E_{2}, \mathrm{MPa}$ & $G_{12}, \mathrm{MPa}$ & $v_{12}$ \\
\hline 150 & 152.9 & 7.3 & 4.4 & 0.32 \\
175 & 153.9 & 7.2 & 3.4 & 0.32 \\
200 & 147.0 & 5.5 & 2.6 & 0.32 \\
\hline
\end{tabular}




\section{Test Procedures and Equipment}

Before testing, all specimens were dried for at least $15 \mathrm{~h}$ at $110^{\circ} \mathrm{C}$ in a convection oven. After drying, the specimens were stored in a dissector until the start of the testing. Any thermal exposure prior to the start of testing did not affect the data since the specimen was rejuvenated immediately prior to testing (see following sections). After each test sequence, the specimens were inspected for matrix cracks along their edges with an optical microscope.

Three test temperatures selected for study were 195,200 , and $212^{\circ} \mathrm{C}$. The two lower temperatures provided data near the upper use temperature of the material. The highest test temperature was selected to ensure that the aging to a point near equilibrium would occur within a reasonable amount of time. For IM7/8320, which has a $T_{g}$ of $221.3^{\circ} \mathrm{C}$, the aging time to equilibrium based upon $\mathrm{Eq}$ 5 would vary from $35.2 \mathrm{~h}$ for the upper test temperature of $212^{\circ} \mathrm{C}$ to $17.3 \times 10^{6} \mathrm{~h}$ for the lower test temperature of $195^{\circ} \mathrm{C}$.

All of the creep tests were performed in convection ovens equipped with digital controllers. Temperature monitoring and feedback thermocouples were placed near the test section. Thermal apparent strain was corrected for by using the compensating gage technique [9]. Load was applied through a dead-weight cantilever arm and reacted at a point outside the test chamber. Mechanical wedge grips held the specimen during the loaded or creep segments. Stress was calculated based upon the applied load and the specimen cross section measured before testing. During the unloaded or recovery segments, the lower grip was released using a remote cable and pulley system. This mechanism allowed the test chamber to remain closed during the entire test sequence.

Strain in the gage section was measured with high-temperature foil strain gages applied in the center of the specimen. The gage type was Micro-Measurement WK-00-250BG-350 with the Mbond-600gage adhesive. Proper selection of gage type and adhesive gave coefficient of thermal expansion match and stability at elevated temperatures.

For the $[90]_{12}$ specimens, the average measurement of two backto-back gages, aligned longitudinally, were used to compute. $S_{22}$. For the $[ \pm 45]_{2 \mathrm{~s}}$ specimens, the average measurement in each direction of four gages, two back-to-back aligned longitudinally and two back-to-back aligned transversely, were used to compute $S_{66}$. The compliance terms for these specimens are as follows

$$
\begin{gathered}
S_{22}(t)=\frac{\epsilon_{x}(t)}{\sigma_{x}} \\
S_{66}(t)=\frac{2 \epsilon_{x}(t)\left[1-\epsilon_{y}(t) / \epsilon_{x}(t)\right]}{\sigma_{x}}
\end{gathered}
$$

Each gage formed a quarter-bridge circuit. Thermal strain compensation was accomplished during data reduction. Commercially available instrumentation provided bridge completion, excitation, and signal conditioning. A personal computer equipped with a 12 bit $A / D$ board converted and stored the data.

Creep Compliance and Physical Aging-A procedure for measuring creep compliance, as described in Struik [1], was used for all tests. This procedure consisted of a sequence of isothermal creep and recovery tests. Each creep period was short in comparison with the previous aging time. During the aging process, the short-term creep tests required a constant load. After each creep segment, the specimen was unloaded and allowed to recover until the start of the next creep test. Figure 2 is a schematic of this procedure. Table 2 provides the temperatures and applied axial stress for each test sequence.

As part of the test procedure, immediately before the start of any physical aging test sequence, the specimen was held at $235^{\circ} \mathrm{C}$ (approximately 15 degrees above it's $T_{g}$ ) for $15 \mathrm{~min}$ and then rapidly quenched with compressed air to the test temperature. This excursion above the $T_{g}$ rejuvenates the specimen and erases any prior physical aging history. After reaching the test temperature during the quench, the aging time started.

The ratio between the prior aging time and each creep test time was at least 10:1 for the sequenced tests. Using this ratio and the sequencing procedure, up to seven creep tests were conducted during an aging time of approximately $120 \mathrm{~h}$. One point of concern for such sequenced tests is the effects of repeated loading on the aging process. To check out such loading effects, sequenced creep tests were run for $27 \mathrm{~h}$ aging (three loading segments). The 27-h sequenced compliance data was then compared to data from a specimen with the same 27 -h aging time but without the intermediate creep segments.

During the creep recovery segments of each aging test sequence, the specimen had no load on it. The recovery times were long

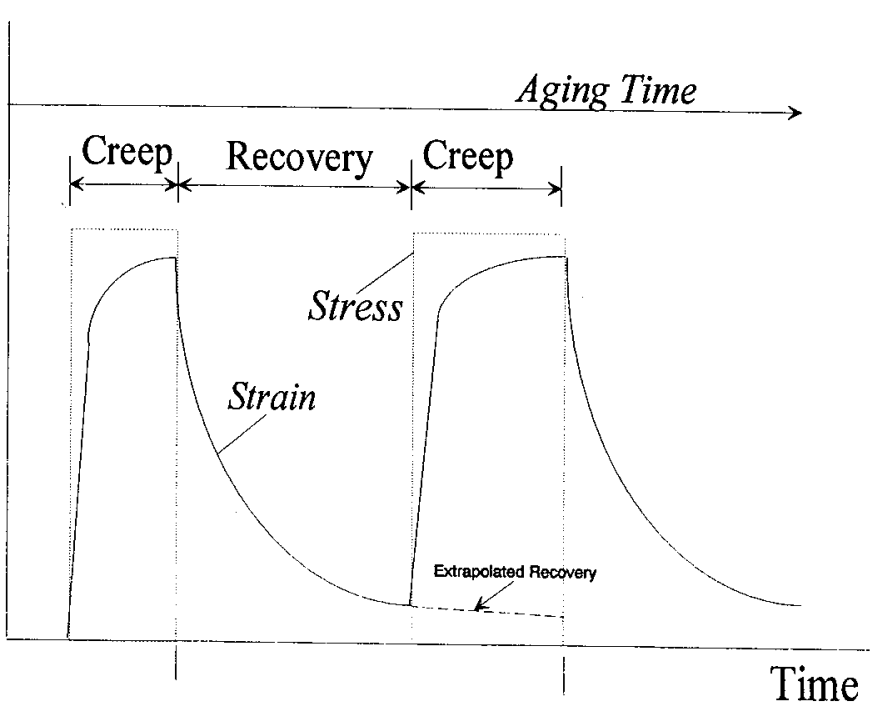

FIG. 2-Schematic of creep/recovery sequence for an aging test.

TABLE 2-Applied axial stress, master curve parameters, and shift rates for the transverse and shear tests. The $\mathrm{S}_{22}$ and $\mathrm{S}_{66}$ data are from the $[90]_{12}$ and $[ \pm 45]_{2 s}$ specimens, respectively.

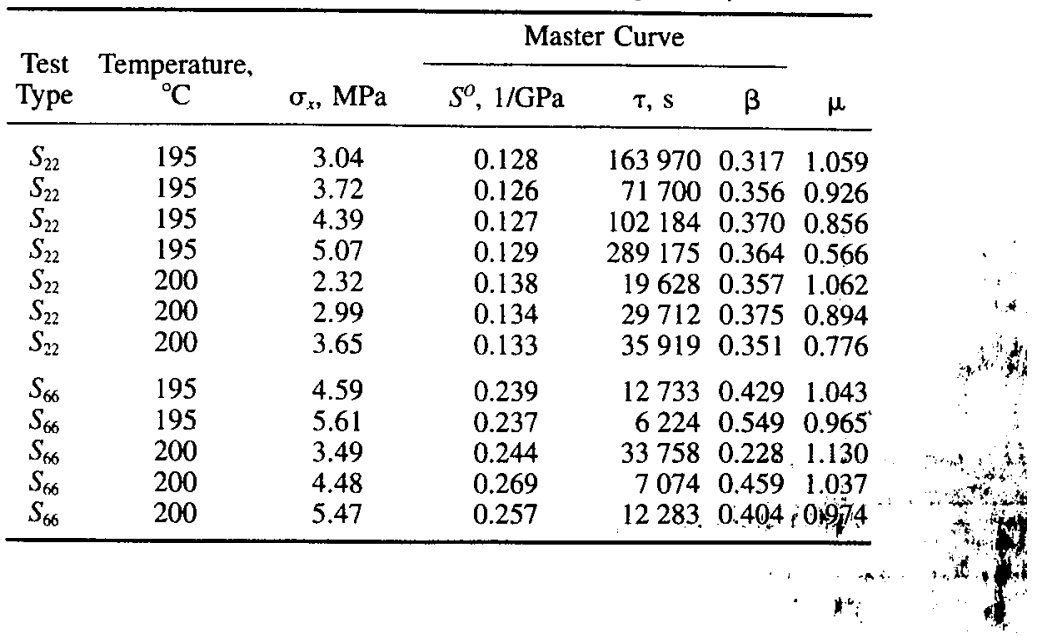


enough to allow for nearly complete strain recovery. However, to account for any remaining strain, the strain measured in the creep segment was corrected by subtracting the extrapolated prior recovery curve from the creep curve, as shown in Fig. 2. To further investigate the effects of short versus long recovery times, two sets of sequenced creep/recovery tests were run with identical aging times but different creep/recovery times. Figure 3 is a schematic illustrating these two test schedules.

Thermoreversibility of Physical Aging-Due to the supposed thermoreversibility of the physical aging process, a single specimen could be used for several aging tests as long as the rejuvenation occurred before each test sequence. This reversibility was verified by measuring creep compliance from a single specimen loaded several times with a rejuvenation sequence before each loading.

Determination of Applied Stresses-For linear viscoelastic behavior, it was assumed that superposition and proportionality would hold. Given an initial state of stress $\sigma^{\prime}$ applied for a time $t$ and an additional stress $\sigma^{\prime \prime}$ applied at a time $t_{1}$, superposition implies that

$$
\epsilon\left[\sigma^{\prime}(t)+\sigma^{\prime \prime}\left(t-t_{1}\right)\right]=\epsilon\left[\sigma^{\prime}(t)\right]+\epsilon\left[\sigma^{\prime \prime}\left(t-t_{1}\right)\right]
$$

while proportionality states that for an applied stress $\sigma$, the strain in a material at any other stress state is found using

$$
\epsilon[c \sigma(t)]=c \epsilon[\sigma(t)] \text { where } c=\text { constant }
$$

Creep and creep/recovery data from several loadings at different stress levels provided data for checking superposition. Proportionality was checked by plotting isothermal, isochronous stiffness versus applied stress for a specimen that was repeatedly rejuvenated, quenched, and loaded at increasingly higher stress levels. The supposed transition from linear to nonlinear behavior should be evident by a slope change in the data.

\section{Results: Tensile Creep Compliance}

Results from the experiments illustrate the effects of stress and physical aging on the elevated temperature creep compliance of IM7/8320. Superposition, proportionality, sequencing effects, load duration effects, stress overload effects, aging to equilibrium, aging shift factors, and aging shift rates were measured and quantified.

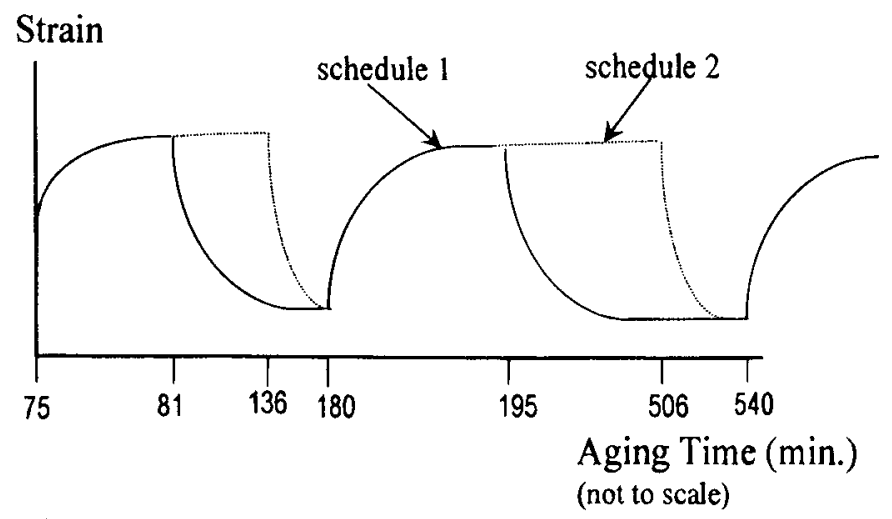

FIG. 3-Schematic of creep/recovery schedules used in tests to assess the effects of load duration.
Superposition-For linear viscoelastic behavior, superposition should hold. For the creep test, superposition states that the recovery strain can be found using Eqs 2 and 7 such that

$$
\epsilon\left(t^{*}\right)=\sigma S^{o}\left\{e^{(t / \tau)^{\beta}}-e^{\left(\left(t-t_{1}\right) / \tau\right)^{\beta}}\right\}
$$

where $\sigma$ is the constant stress, $t_{1}$ is the time of load removal, $t^{*}$ is the time during the recovery segment, and $S^{o}, \tau$, and $\beta$ are the constants from the measured creep compliance curve. Equation 9 was used to predict the recovery of a $[90]_{12}$ transverse specimen loaded at sequentially higher load levels. The specimen was rejuvenated before each test and aged for exactly 30 min before loading. Figure 4 provides the predicted strain/time curves along with the measured data.

Comparison of test and prediction for the recovery segments indicates that only the recovery test data at the lowest load level was accurately predicted by superposition. However, the predicted strain rate during recovery corresponded well with the measured value for all load levels tested.

Proportionality-One of the clearest ways to check if proportionality holds for creep tests is to plot the isochronous stiffness versus applied stress levels at a constant temperature. The stiffness values were calculated by inverting the measured creep compliance data for any given time during the loaded segment of the test. Therefore, it should be noted that the stiffness values were not true stiffness as calculated from stress-relaxation tests. Unproportional behavior will be evident by deviations of the data from horizontal lines as stress increases. Figures 5 through 8 give examples of such data.

Figures 5 and 6 indicate little or no unproportional behavior as a function of applied stress, whereas Figs. 7 and 8 show a transition to unproportional behavior. Comparing Figs. 5 through 7 shows that these deviations from linearity became more apparent as the test temperature increased. The stress levels chosen for the aging experiments are indicated on these figures. Using the isochronous stiffness data from Figs. 5 through 8, the aging test stress levels were chosen to correspond to the range where little or no deviation from proportionality was observed.

Sequencing Effects-To ensure that the sequencing procedures of the momentary creep tests worked, checks were made using different specimens and test temperatures. For the sequencing to work properly, any creep compliance data from the sequenced

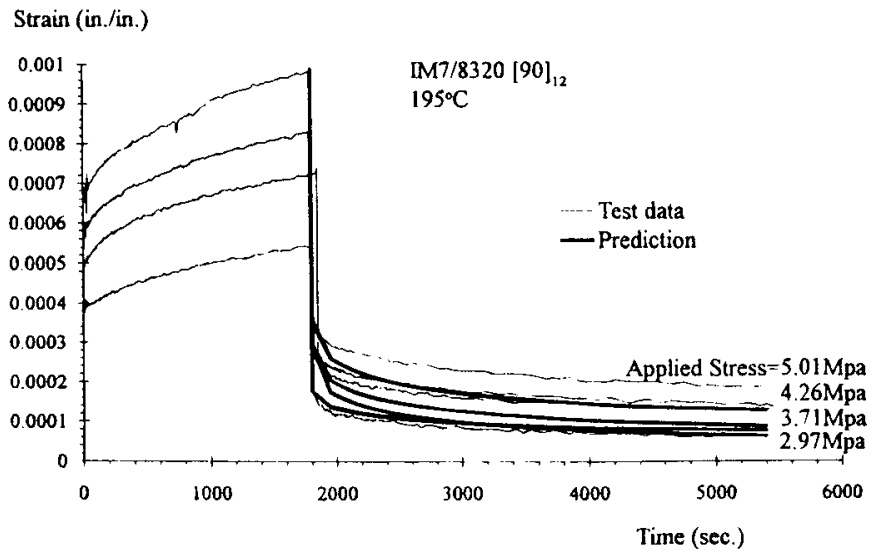

FIG. 4-Recovery data versus prediction made using Boltzman superposition principle. 
IM7/8320 [90 $]_{12}$

$195^{\circ} \mathrm{C}$

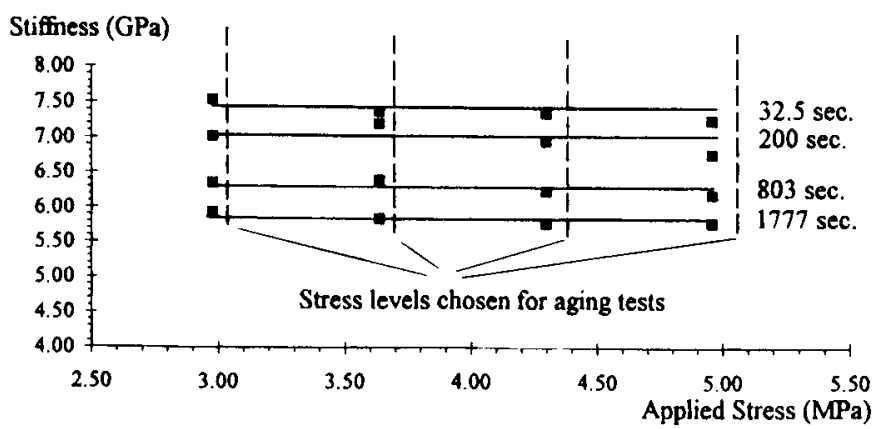

FIG. 5-Proportionality check using creep data of transverse specimens tested at $195^{\circ} \mathrm{C}$.

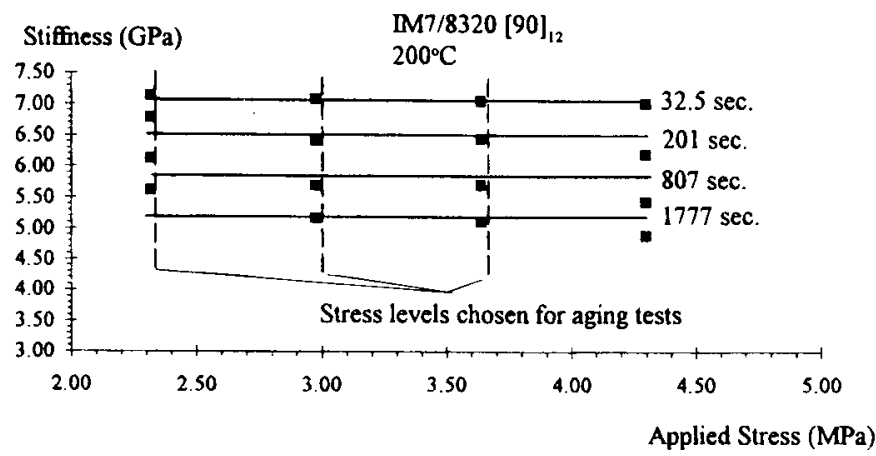

FIG. 6-Proportionality check using creep data of transverse specimens tested at $200^{\circ} \mathrm{C}$

IM7/8320 $[90]_{12}$

$212^{\circ} \mathrm{C}$

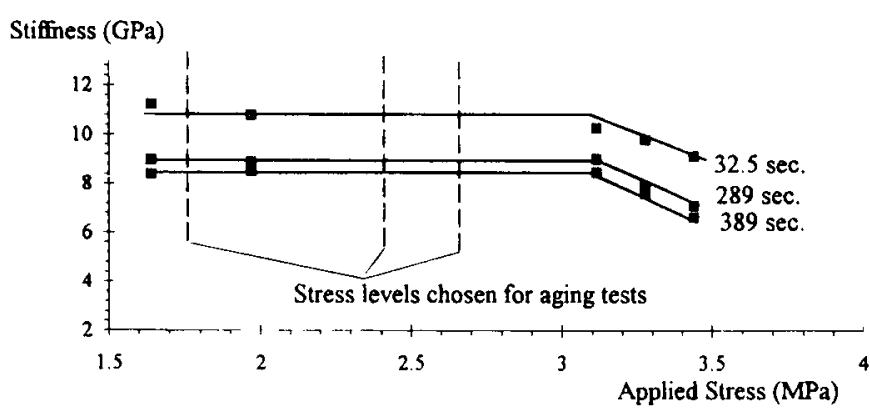

FIG. 7-Proportionality check using creep data of transverse specimens tested at $212^{\circ} \mathrm{C}$.

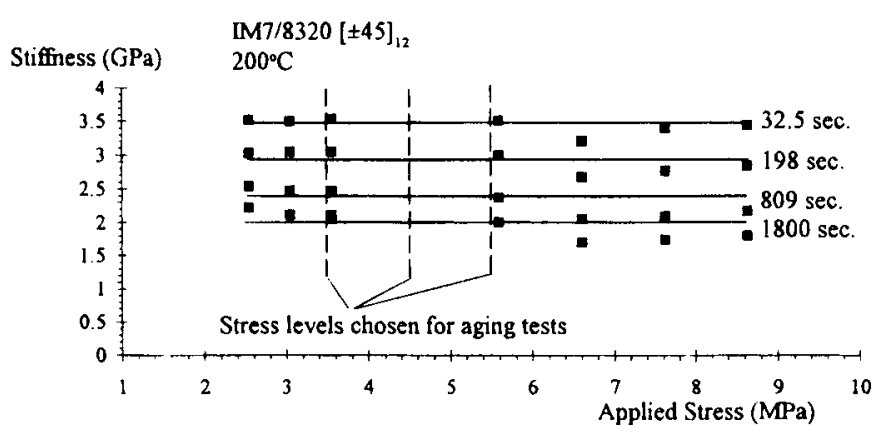

FIG. 8-Proportionality check using creep data of shear specimens tested at $200^{\circ} \mathrm{C}$. creep/aging experiment should coincide with an unsequenced creep compliance measurement taken at the same aging time. Figure 9 shows an example of this behavior for a $[ \pm 45]_{2 s}$ specimen loaded at $1,3,9$, and $27 \mathrm{~h}$ of aging time versus the same specimen loaded only at $27 \mathrm{~h}$ aging. Both tests included a rejuvenation period before the start of aging.

Examination of the compliance data in Fig. 9 indicates that the sequenced tests produced equivalent $27 \mathrm{~h}$ compliance data compared to the unsequenced test. This demonstrates that the short loading periods of the sequenced tests did not measurably effect the data, thereby ensuring that the sequenced test procedure gave a true measure of physical aging.

Load Duration Effects-The momentary creep compliance measured during the aging tests was essentially a "snapshot" of the aging process. This required that the duration of a creep segment be kept much lower than the cumulative aging time, thereby ensuring minimal influence on the aging process moreover allowing for a sufficient time for strain recovery to occur. The data from all the strain recovery segments of the sequenced creep compliance measurements indicated that near complete recovery took place. As suggested by Struik [1], the unrecovered strain was subtracted from the total strain during the following creep segment. The unrecovered strain was computed using a linear extrapolation of the steady-state portion of the creep recovery curve. Figure 2 schematically illustrates this procedure.

To explore the effects of load duration on creep compliance, two different test schedules were followed. Figure 3 gives the times for the creep and recovery segments of these two test schedules. The test sequence with the short creep segments was designated Schedule 1 and the test sequence with the long creep segments was designated Schedule 2. Figure 10 shows the measured effects on creep compliance due to loading according to these schedules.

Figure 10 clearly illustrates that the extended creep times used in Schedule 2 produce different creep compliance curves than the data from Schedule 1. The differences are due to the additional creep occurring during the extended load time. If the curves from both schedules are fit with $\mathrm{Eq} 2$, different parameters will result. However, the effects of aging ( $\log$ a shifts) are apparent using either test schedule. For material systems unlike IM7/8320 that would not exhibit such rapid strain recovery, the effects of extended load periods would become even more pronounced.

Stress Overload Effects-As outlined by Struik [1], a momentary stress overload during a normal sequenced creep compliance test

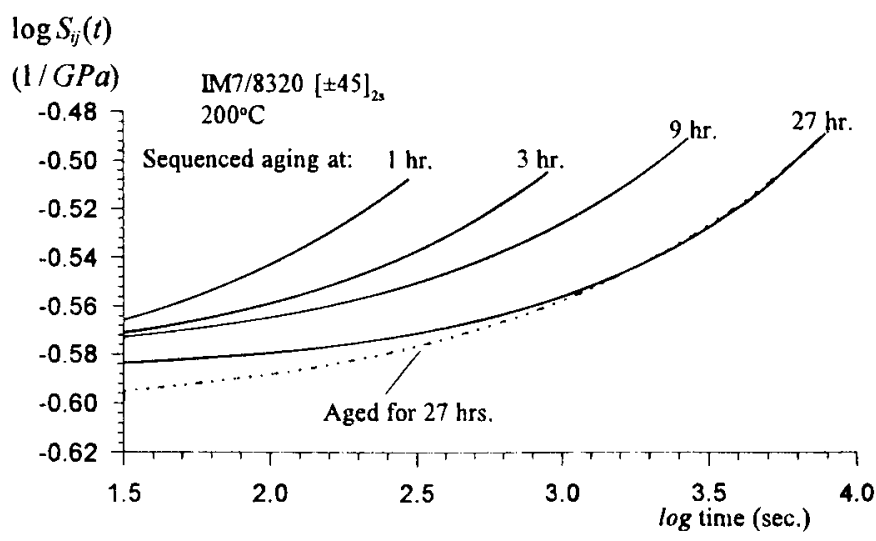

FIG. 9-Comparison of sequenced versus unsequenced creep compliance test data. 


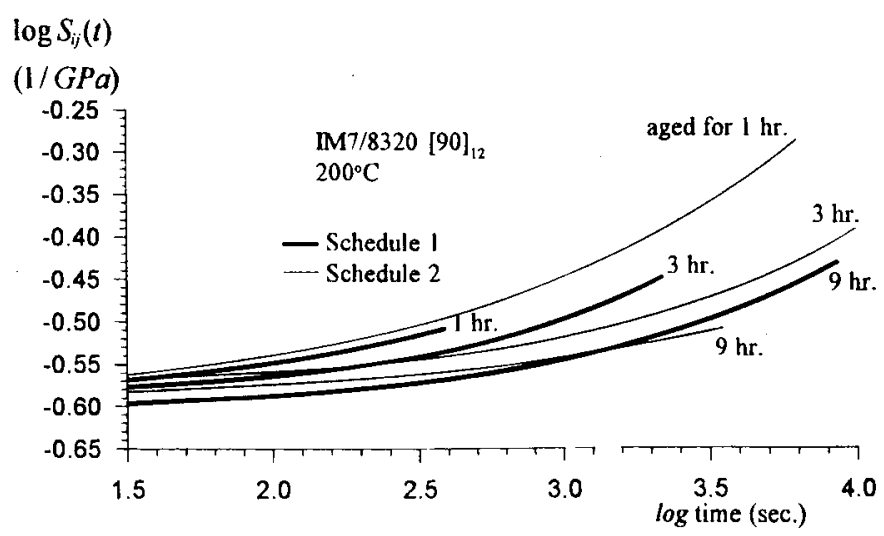

FIG. 10-Test data showing the effects of extended load periods during sequenced creep compliance testing.

for aging may upset the aging process in the material. He states that if this overload is sufficiently high, the material may apparently deage. To explore this idea, a sequenced creep compliance test was run on a $[90]_{12}$ transverse specimen at $200^{\circ} \mathrm{C}$. Figure 11 shows the load/time history imposed on this specimen. For the first three segments of the sequenced test, the load was kept at a constant. The aging time for these segments was 1,3 , and $9 \mathrm{~h}$. The forth segment occurred at $24 \mathrm{~h}$ and consisted of an overload that was approximately 1.7 times the previous load. The initial load was then used for the subsequent segments. To capture any immediate or transient effects on the aging process, the segments after the overload started at $1,3,9$, and $26 \mathrm{~h}$ after the overload or 26,28 , 34 , and $51 \mathrm{~h}$ total aging time, respectively.

Figure $12 a$ gives the resulting creep compliance curves. The 1-, 3-, 9-, and 24-h aging time segments collapsed to form one momentary master curve referenced to the 1-h curve. The 26-, 28-, 34-, and 51-h aging time segments collapsed to form a momentary master curve referenced to the 26-h curve. Apparently these curves are distinct in both shape and location.

Figure $12 b$ gives the resulting shift factors. All the factors are first plotted using the 1-h curve as a reference for all the data. As shown in the Fig. 12b, a slight disruption of the data occurs after the overload. However, deviations from linearity of the shift rate $\mu$ are not significant. Fitting all the data gave a shift rate of .90 . If just the post-overload shift factors are plotted referenced to the 26-h test, the shift rate decreased to .30 .

Aging to Equilibrium-An experimental study on the ability to age the material to a state near equilibrium was performed using

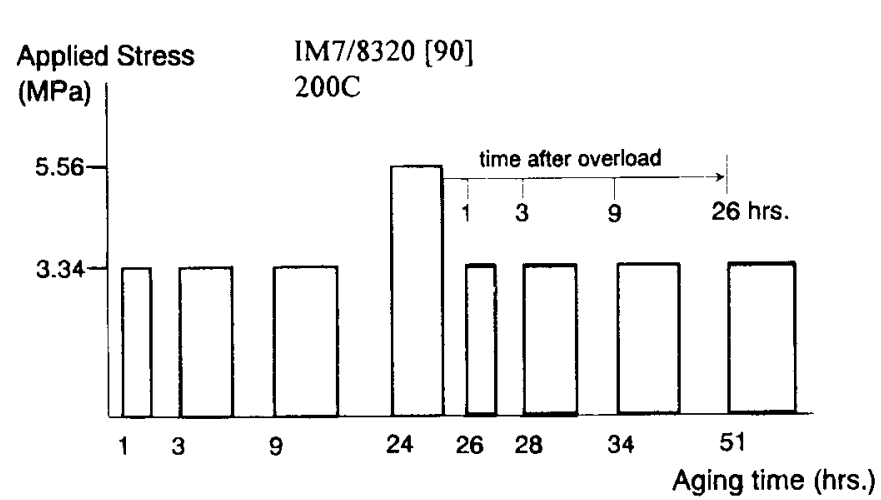

FIG. 11-Loading schedule for the stress overload test.

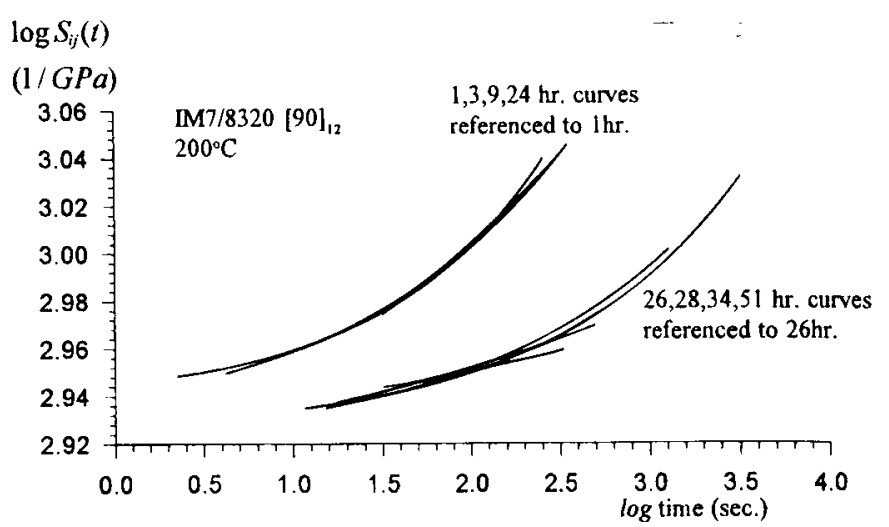

FIG. 12a-Measured creep compliance curves prior to and after a stress overload.

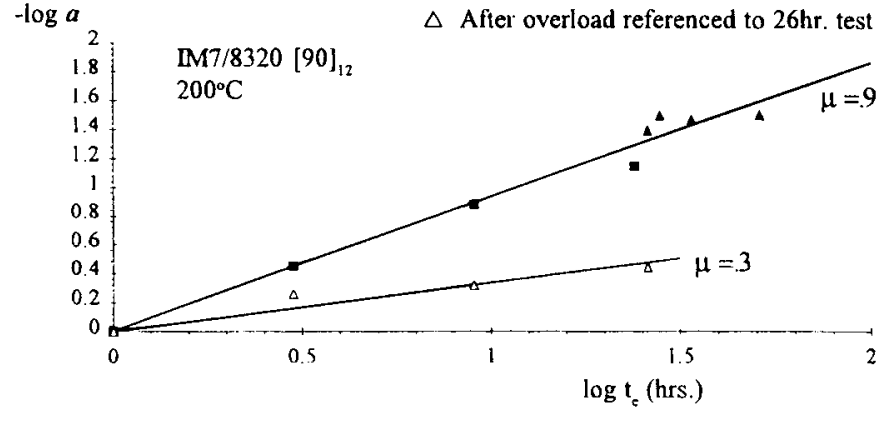
load

FIG. 12b-Measured aging shift factors prior to and after a stress over-

$S_{22}$ specimens tested at $212^{\circ} \mathrm{C}$ and four stress levels. Figures 13 and 14 give the shift factors for these tests. Shift factors used in Fig. 13 are from using only the horizontal time shift to create the master curves. Figure 14 shows results from the same tests but with the time shifts computed after a vertical compliance shift. A bilinear least squares fit to the data was computed for all four data sets and is shown in Figs. 13 and 14. The break in these bilinear fits was made at the 3 - $\mathrm{h}$ aging point. Additional data points before and after this $3-\mathrm{h}$ point would further define these break points. The 3-h data point for the $385 \mathrm{psi}(2654.5 \mathrm{kPa})$ data set was not included in the curve fit due to excessive scatter.

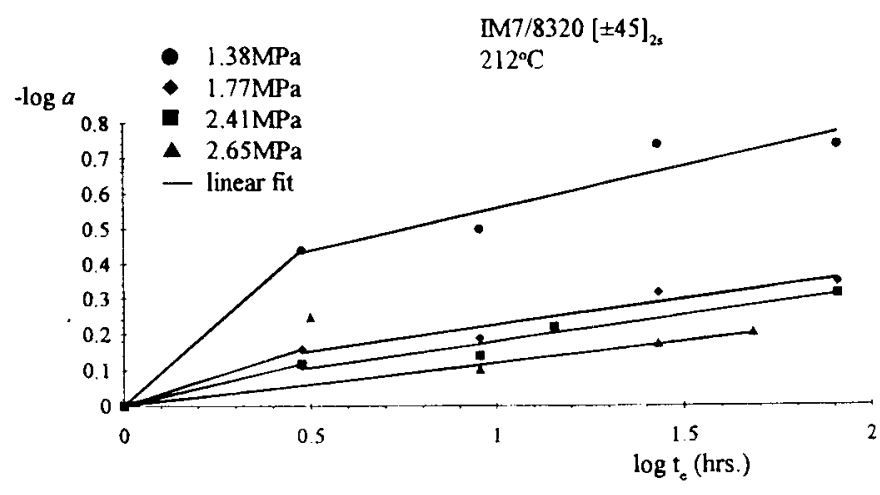

FIG. 13-Measured shift factors form an aging to equilibrium test, found using horizontal (time) shifts only. 


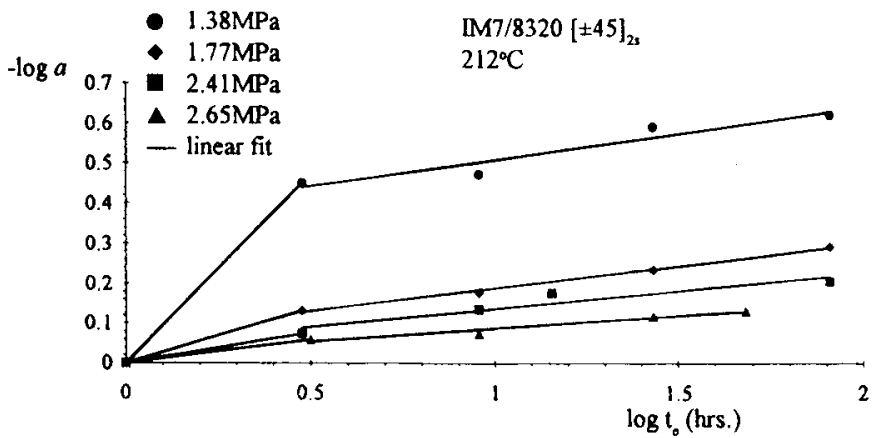

FIG. 14-Measured shift factors form an aging to equilibrium test, found using horizontal (time) and vertical (compliance) shifts.

A comparison of Figs. 13 and 14 show that inclusion of the vertical shift in the collapse of the data to form the master curve may decrease the scatter of the aging factors. However, the general trends in the data do not change by including this vertical shift.

Aging Shift Factors-Given a set of momentary creep compliance curves, as shown in Fig. 1, the time shift factors $(\log$ a) for each curve were measured by referencing all curves to the first (1-h) creep compliance curve. To facilitate computation of these shift factors, each curve was fit with the three-parameter model shown in Eq 2. The smooth curves generated from these fits were shifted along the time axis until they coincided with the reference curve and therefore created a momentary master curve. The master curve data was also fit with $\mathrm{Eq} 2$. Table 2 gives the three parameters from the analysis of the master curve data for the 195 and $200^{\circ} \mathrm{C}$ sequenced tests. Figures 15 through 18 provide the master curves for $S_{22}$ and $S_{66}$.

For many of the tests, a vertical or compliance shift would facilitate the collapse of the data. However, the time shifts due to aging were of primary importance in this study; therefore, all the shift factor and shift rate curves shown in the results were found using the time $(\log$ a) shifts only, unless otherwise noted.

Figures 19 through 22 present the time shifts versus aging time. In these figures, a linear least squares fit to each data set is also shown. Each linear fit represents a different applied stress level. Figures 19 and 20 are the $S_{22}$ data at 195 and $200^{\circ} \mathrm{C}$, respectively. Figures 21 and 22 are the $S_{66}$ data at 195 and $200^{\circ} \mathrm{C}$, respectively. All the data presented in these figures have a data point at the origin.

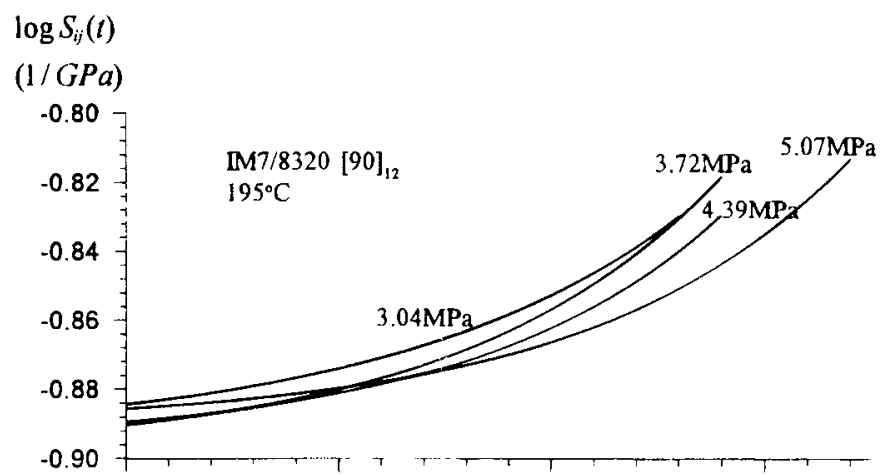

FIG. 15-Momentary master curves for a transverse specimen at $195^{\circ} \mathrm{C}$ and various applied stress levels.

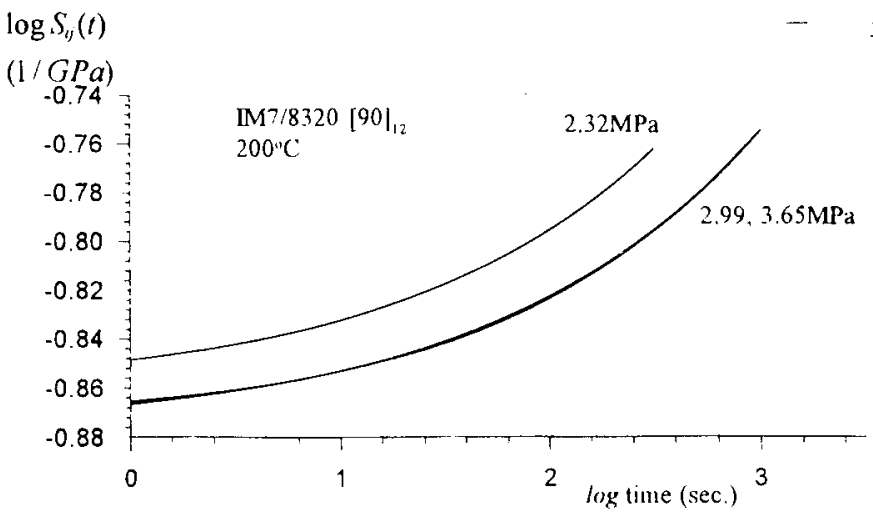

FIG. 16-Momentary master curves for a transverse specimen at $200^{\circ} \mathrm{C}$ and various applied stress levels.

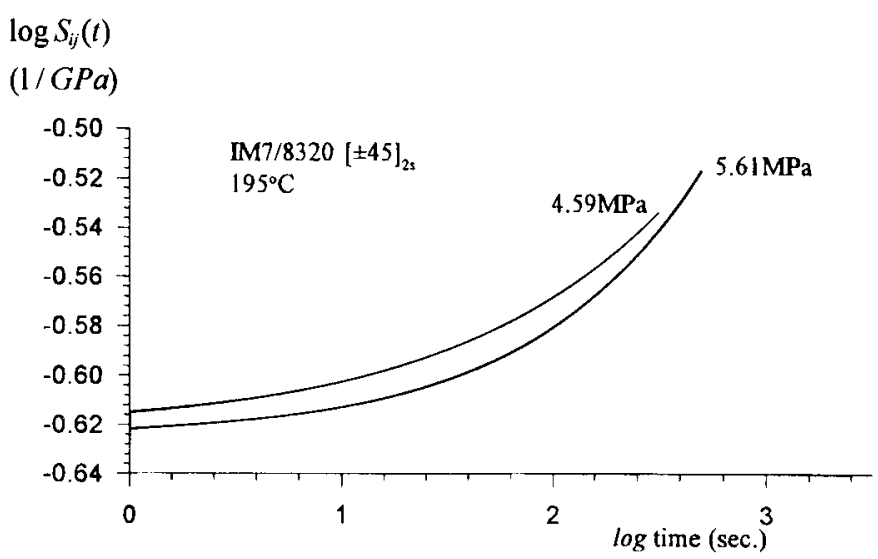

FIG. 17-Momentary master curves for a shear specimen at $195^{\circ} \mathrm{C}$ and various applied stress levels.

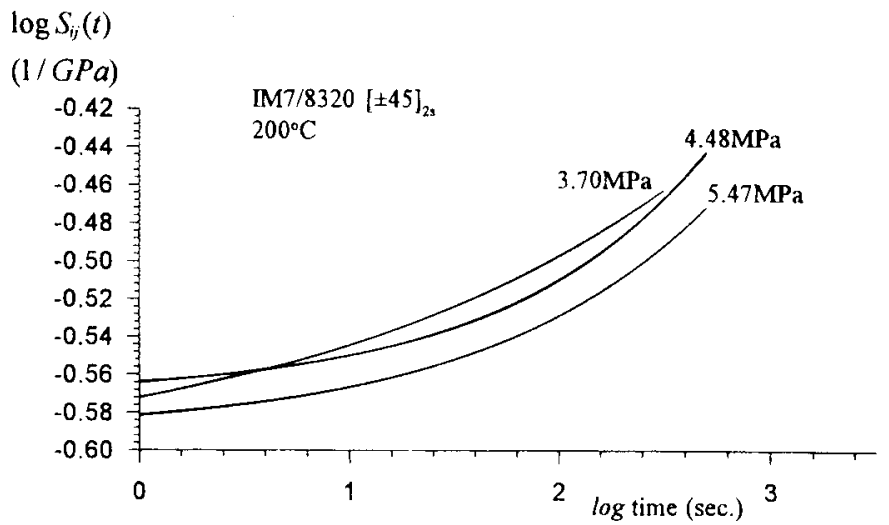

FIG. 18-Momentary master curves for a shear specimen at $200^{\circ} \mathrm{C}$ and various applied stress levels.

Aging Shift Rates-The aging shift rates for all tests were computed by using linear least squares fits to the aging shift factors. Tables 2 and 3 give the computed shift rates. Figure 23 is a plot of these shift rates as a function of applied axial stress. The $S_{66}$ data at 195 and $200^{\circ} \mathrm{C}$ collapsed and was fit with one curve. The $S_{22}$ data sets for each temperature were separate and fit with their own curves. The shift rates shown in Fig. 23 for the $S_{22}$ specimen at $212^{\circ} \mathrm{C}$ are values from the secondary slope of the bilinear fit. 


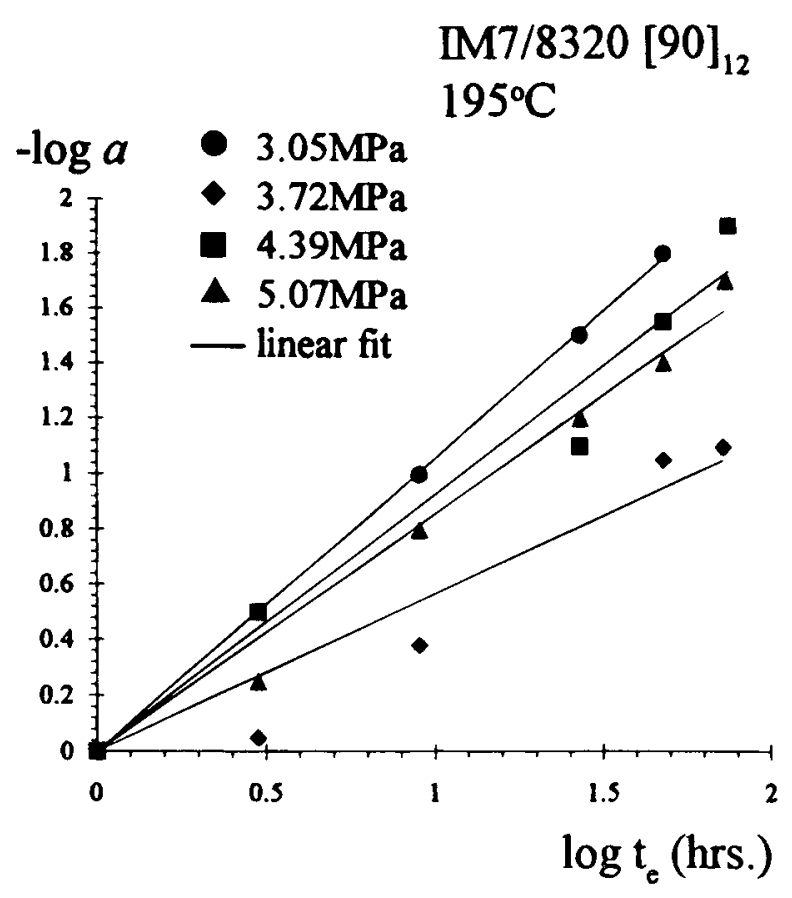

FIG. 19-Measured aging shift factors for transverse specimens at $195^{\circ} \mathrm{C}$ and various stress levels.

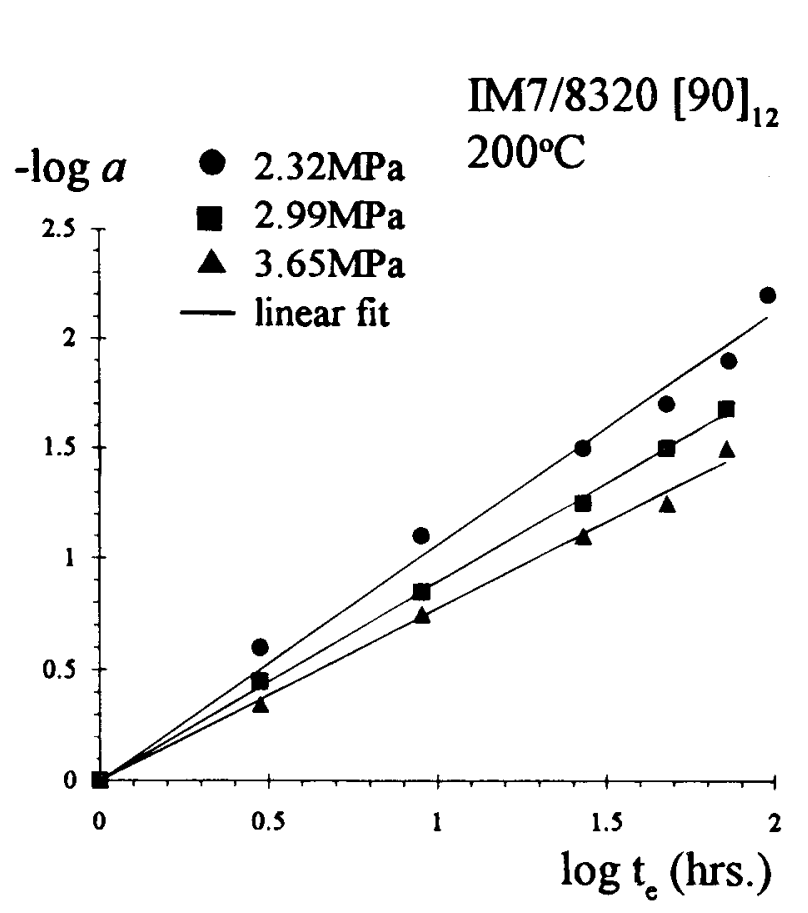

FIG. 20-Measured aging shift factors for transverse specimens at $200^{\circ} \mathrm{C}$ and various stress levels.

These rates were computed using shift factors found from the aging time shift to the data.

Figure 24 uses the same shift rate data as in Fig. 23, but shows it as a function of ply stress for the $S_{22}$ and $S_{66}$ specimens. The transverse stress $\sigma_{22}$ in the $90^{\circ}$ ply of the $S_{22}$ specimen is equal to the applied axial stress. The shear stress $\sigma_{12}$ in a $45^{\circ}$ ply of the $S_{66}$ specimen has a magnitude equal to one half of the applied axial stress. The $195^{\circ} \mathrm{C}, S_{66}$ specimen tested at $960 \mathrm{psi}(6619 \mathrm{kPa})$

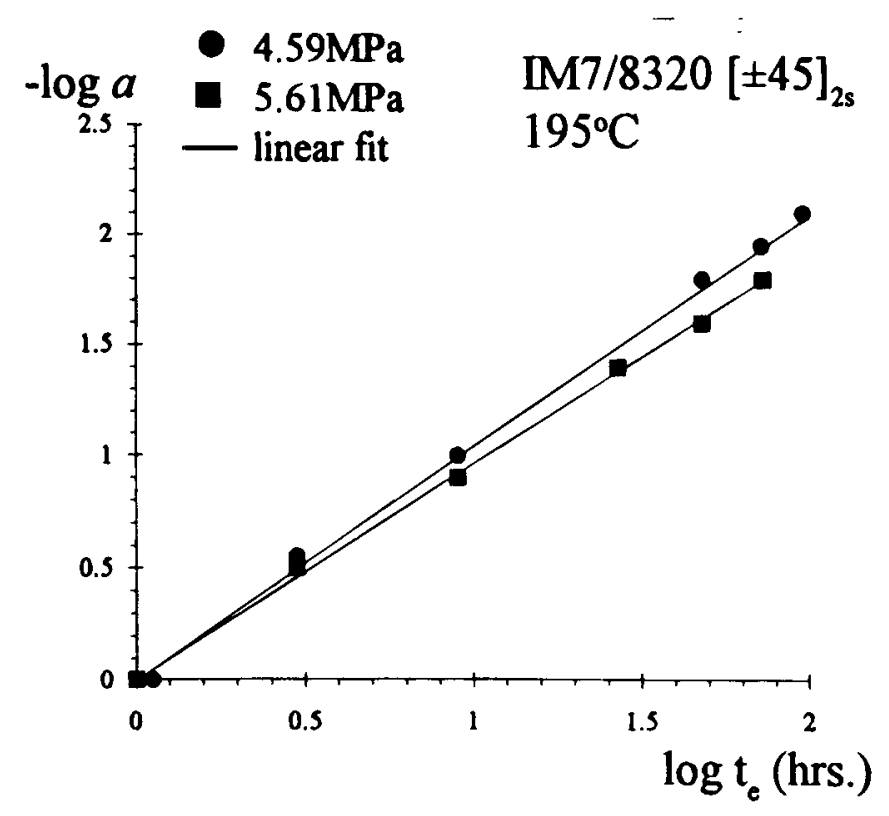

FIG. 21-Measured aging shift factors for shear specimens at $195^{\circ} \mathrm{C}$ and various stress levels.

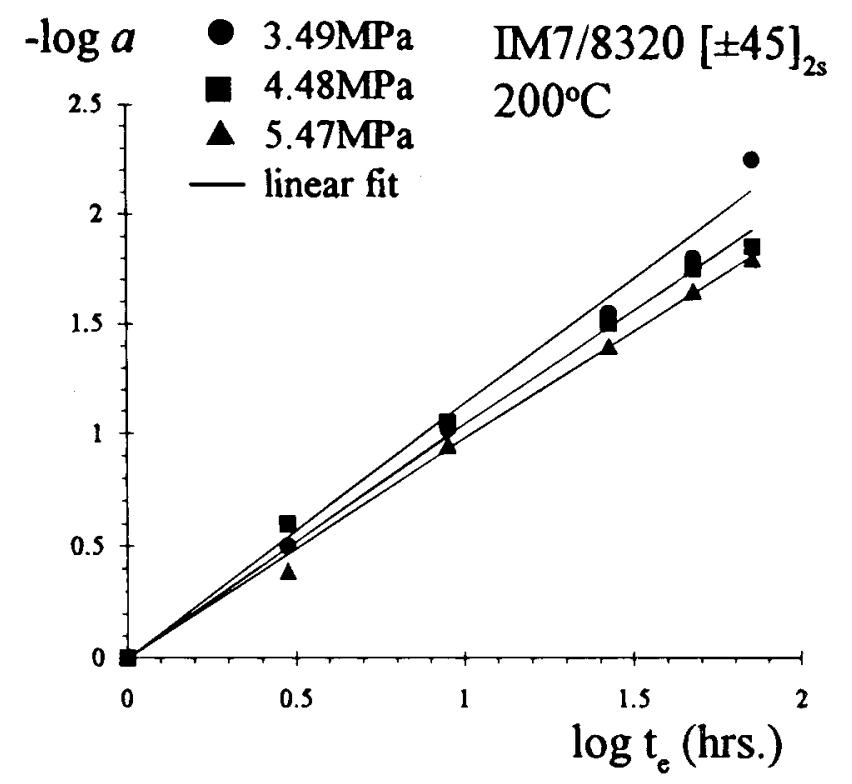

FIG. 22-Measured aging shift factors for shear specimens at $200^{\circ} \mathrm{C}$ and various stress levels.

TABLE 3-Aging shift rates for the secondary slope of equilibrium

\begin{tabular}{ccc}
\multicolumn{3}{c}{\begin{tabular}{c} 
aging tests. \\
\hline
\end{tabular}$S_{22}$ at $212^{\circ} \mathrm{C}$} \\
\hline$\sigma_{x}, \mathrm{psi}$ & $\mu^{a}$ & $\mu^{b}$ \\
\hline 200 & 0.132 & 0.239 \\
256 & 0.114 & 0.147 \\
350 & 0.091 & 0.147 \\
385 & 0.065 & 0.147 \\
\hline
\end{tabular}

${ }^{a} \mu^{*}=$ horizontal shift rate using horizontal and vertical shift.

${ }_{b} \mu=$ horizontal shift rate using horizontal shift only. 


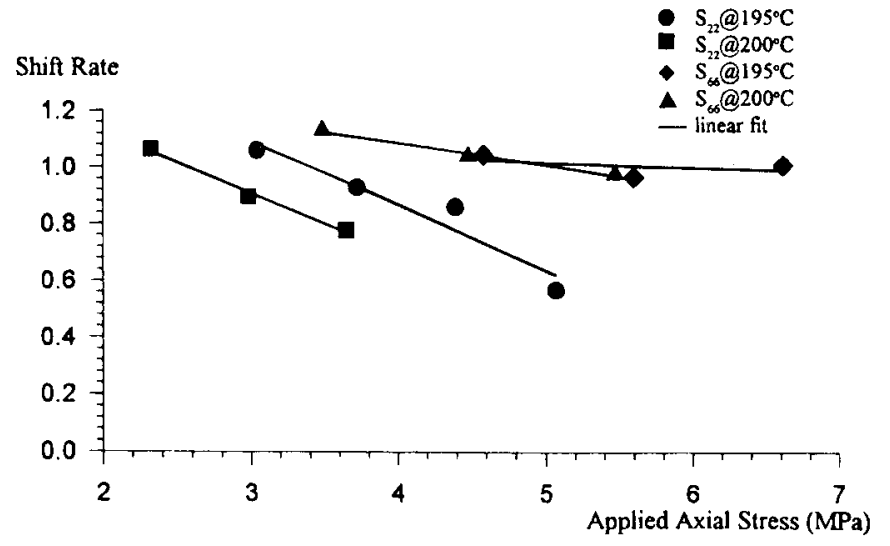

FIG. 23-Measured aging shift rates for all tests versus the applied stress.

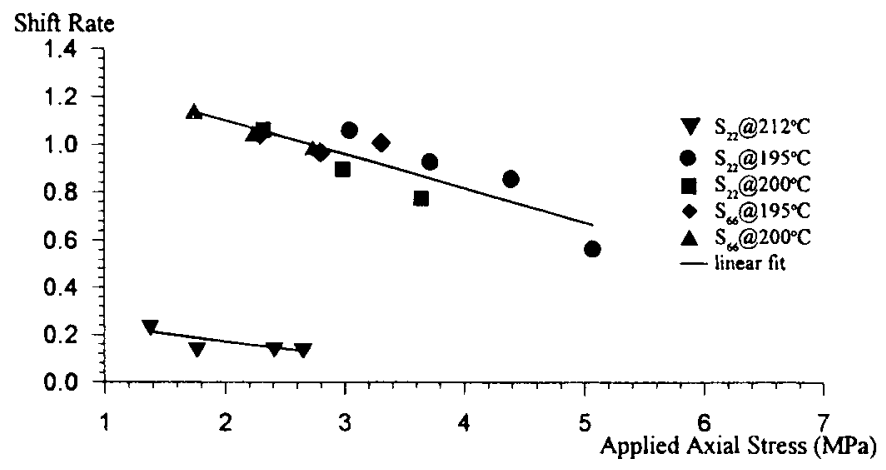

FIG. 24-Measured aging shift rates for all tests versus the resultant ply stress.

was the only specimen in which damage (transverse matrix cracks) was detected during post-test inspection. This data point was not used in the curve fit of Fig. 23 or 24.

\section{Discussion and Summary: Tensile Creep Compliance}

Linear viscoelasticity assumes that both proportionality and superposition hold. Data from Figs. 4 through 8 reveals the difficulty in checking these concepts for elevated temperature PMCs. The inability of superposition to accurately predict strain recovery for all stress levels indicates some stress-level dependency. However, the proportionality data suggests that there should be a range of stress levels available for testing in the linear viscoelastic range. For temperatures sufficiently close to the $T_{g}$, PMCs may exhibit nonlinear behavior in the matrix constituent. The lack of a sharp transition from linear to nonlinear behavior would suggest that if test stress levels are low, linear viscoelasticity may provide a first level approximation when developing test and analysis procedures.

The sequenced creep/recovery procedures seem to work well for characterizing physical aging in PMCs. It appears necessary to keep the ratio between aging time and test time to a value of ten or greater to ensure that loading has a minimum influence on aging and that the material has sufficient time to undergo strain recovery.

The tests on the $S_{22}$ specimen at $212^{\circ} \mathrm{C}$ indicated that if the temperature was sufficiently close to the $T_{g}$, a change in the aging shift rate may occur. Figures 13 and 14 indicate that these breaks in shift rate, or time-to-equilibrium, are relatively independent of applied stress level. This result has important implications for long-term testing. The aging towards equilibrium behavior for the IM7/8320 material was similar to the results achieved by Lee and McKenna [7] for epoxy glasses.

Momentary master curves do not reveal a clear trend when examined as a function of stress level. Examining Figs. 15 through 18 show that, in general, the master curves will shift to the right along the time axis as stress level increases. However, the data does not appear to lend itself well to a time-stress superposition type of analysis. The plots of shift factor versus aging time given in Figs. 19 through 22 verify the trend in shift factors as a function of applied stress.

Plotting shift rate $\mu$ as a function of applied axial stress, as in Fig. 23, indicates that $\mu$ for the two creep compliance terms have different aging shift rates. The $S_{22}$ term shows a higher dependency on applied axial stress. In addition, the $S_{22}$ data shows some temperature dependency when compared to the $S_{66}$ data. This difference in temperature dependency between the two compliance terms is probably due to the low creep strains of the $S_{22}$ data and the inability of the instrumentation to accurately resolve strains less than 10 $\mu \epsilon$. The $S_{66}$ data gave less scatter at both test temperatures.

Struik [1] has shown that the shift rate $\mu$ of rigid PVC loaded under two separate loads, tensile and shear, will collapse when plotted against applied stress. This stress was assumed to be large and to cause nonlinear creep. He further states that the collapse of the $\mu /$ stress data appears to be independent of test temperature. Plotting the shift rates from Table 2 as a function of ply stress, as in Fig. 24, indicated the data for the $S_{22}$ and $S_{66}$ specimens may collapse into a single data set where the temperature and loadtype dependency are less apparent.

Struik [1] has interpreted this stress-level dependency in polymers as an indication that large stresses produce an erasure or deaging of the material. Lee and McKenna [7] have offered an alternative interpretation of the decrease in shift rate as a function of an increase in the applied stress. They state that "an increase in the amplitude of the stress applied in the physical aging experiment results in a decrease in the shift rate $\mu$ simply because the changes in structure accompanying volume recovery affect the non-linear response differently at large stresses than at small ones." They further state that this does not imply that the structure of the polymer is changed by the application of mechanical loads. At this point, the authors feel that additional data on the composite material is needed to fully validate either of the theories presented.

\section{Acknowledgment}

The authors would like to acknowledge the expert technical laboratory assistance of Ms. Lois White and Mr. Reggie Rose.

\section{References}

[1] Struik, L. C. E., Physical Aging in Amorphous Polymers and Other Materials, Elsevier Scientific Publishing Company, New York, 1978.

[2] Sullivan, J. L., "Creep and Physical Aging of Composites," Composites Science and Technology, Vol. 39, 1990, pp. 207-232.

[3] Hastie, R. L. and Morris, D. H., "The Effect of Physical Aging on the Creep Response of a Thermoplastic Composite," High Temperature and Environmental Effects in Polymer Matrix Composites, ASTM STP 1174, C. Harris and T, Gates, Eds., American Society for Testing and Materials, Philadelphia 1992, pp. 163-185.

[4] Chen-Chi, M., Lee, C., Chang, M., and Tai, N., "Effect of Physical Aging on the Toughness of Carbon Fiber-Reinforced Poly(ether ether ketone) and Poly (phenylene sulfide) Composites. I," Polymer Composites, Vol. 13, No. 6, Dec. 1992, pp. 44l-447. 
[5] Aklonis, J. J., MacKnight, W. J., and Mitchel, S., Introduction to Polymer Viscoelasticity, Wiley-Interscience, New York, 1972.

[6] Press, W. H., Flannery, B. P., Teukolsky, S. A., and Vetterling, W. T., Numerical Recipes, The Art of Scientific Computing, Cambridge University Press, Cambridge CB2 1RP, United Kingdom, 1986.

[7] Lee, A. and McKenna, G. B., "The Physical Ageing Response of an Epoxy Glass Subjected to Large Stresses," Polymer, Vol. 31, March 1990, pp. 423-430.
[8] Gates, T. S., "Matrix Dominated Stress/Strain Behavior in Polymeric Composites: Effects of Hold Time, Nonlinearity, and Rate Dependency," Composite Materials: Testing and Design (Eleventh Volume), ASTM STP 1206, E. T. Camponeschi, Jr., Ed., American Society for Testing and Materials, Philadelphia, 1993, pp. 177-189.

[9] Murry, W. M. and Miller, W. R., The Bonded Electrical Resistance Strain Gage, Oxford University Press, New York, 1992. 
